\title{
An AHP-based Evaluation System of Urban Healthy Growth
}

\author{
Rui Wang ${ }^{1, a, *}$ \\ ${ }^{1}$ School of North China Electric Power University Baoding, Baoding 071000, China. \\ a72770@163.com
}

\begin{abstract}
In recent years, the urban planning theory of Healthy Growth has getting more and more attention, due to the situation of increasing urban population. To better quantify the healthy growth degree of a city, we build an AHP based Evaluation System of Healthy Growth. Firstly, we classify and quantify the impact indicators. Secondly, we make an questionnaire, which can be used to predict the weight of each category when the feedback data tends to infinity. Finally, according to the relative importance of indicators under each category, the judgment matrix is established and the weight of each indicator is obtained. Also, the consistency test is excellent.
\end{abstract}

Keywords: Urban healthy growth, AHP.

\section{Introduction}

Healthy growth is an urban planning and transportation theory that concentrates growth in compact walkable urban center to avoid sprawl. It also advocates transit-oriented , bicycle-friendly land use, including neighborhood schools, complete streets, and mixed-use development with a range of housing choices. There are some other principles for healthy growth, such as fostering distinctive, attractive communities with a strong sense of place and preserving open space, farmland, natural beauty [1]. Our work is to build a realistic, sensible, and useful model to optimize the healthy growth of the city. Considering the insufficient data, we choose Analytic Hierarchy Process (AHP) to build the model.

\section{Model Elaboration}

\subsection{The Establishment of Categories.}

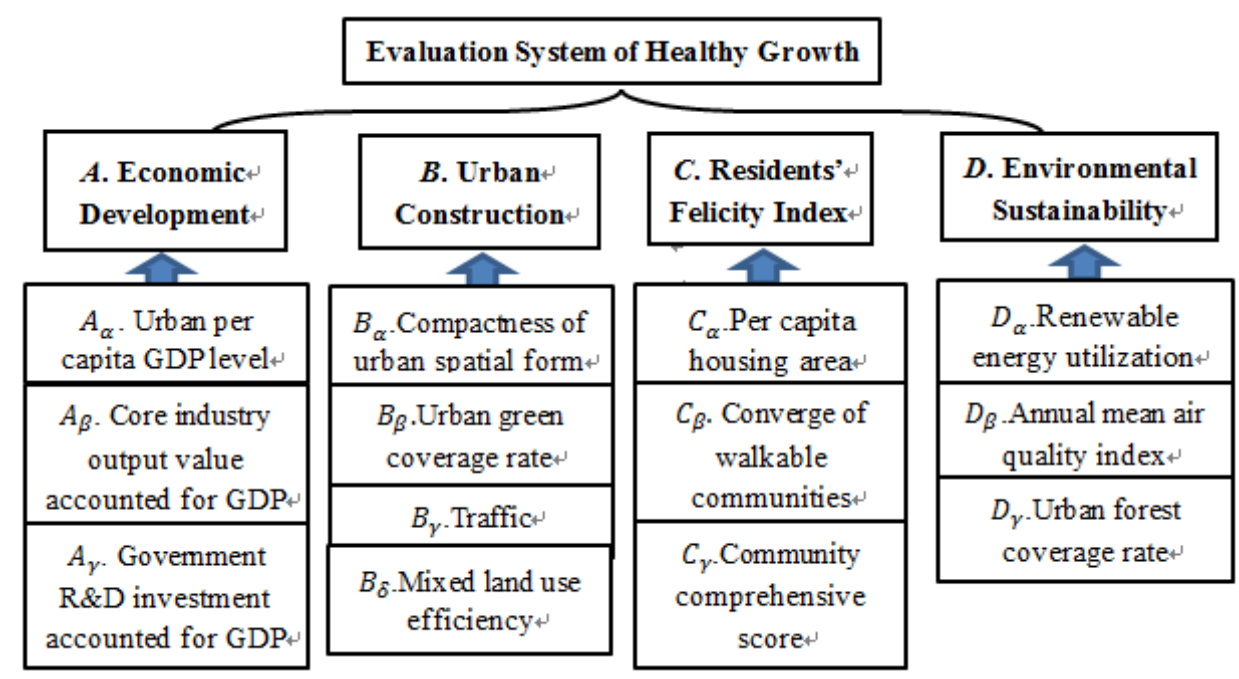

Fig.1 Evaluation indicators of Healthy Growth

The structure of the evaluation system, along with the definition of each category and its internal 
indicators can be displayed in this figure.

\subsection{The Quantification of Indicators.}

In the above figure,

$$
\begin{gathered}
A_{\alpha}=\frac{U G D P}{A G D P} \\
D_{\alpha}=\frac{R}{E}
\end{gathered}
$$

in which,

UGDP is urban per capita GDP.

$A G D P$ is American per capita GDP.

$R$ is the utilization of renewable energy per year.

$E$ is the total amount of energy consumption per year.

$\mathrm{A}_{\beta}, A_{\gamma}, B_{\alpha}, B_{\beta}, B_{\delta}, C_{\alpha}, D_{\beta}, D_{\gamma}$ can be obtained in the relevant information.

$B_{\gamma}$ is used to evaluate the urban traffic, is in relation to the degree of traffic development and traffic congestion.

$C_{\beta}, C_{\gamma}$ is difficult to obtain specific data, are presented in a questionnaire. Our questionnaire is composed of the following parts: 1. Questions pertaining to the public facilities and security situation in the respondents' communities. (50 points) 2. Questions pertaining to the residents' housing feeling of their communities. (50 points) 3. Additional question: Is your community walkable? (Walkable communities are created in part by mixing land uses and taking advantage of compact design, but are activated by smart street design that makes walking not only practical but safe and convenient to enjoy.) The total score of the top two questions get $C_{\gamma}$, the additional question is expected to estimate $C_{\beta}$.

Table 1 Classification criteria of indicators

\begin{tabular}{c|c|c|c|c|c}
\hline Level & $\mathbf{1}$ & $\mathbf{2}$ & $\mathbf{3}$ & $\mathbf{4}$ & $\mathbf{5}$ \\
\hline Indicators & $0 \sim 5$ & $5 \sim 10$ & $10 \sim 15$ & $15 \sim 20$ & $\geq 20$ \\
\hline $\begin{array}{c}\text { Crban per capita GDP level /\% } \\
\text { accounted for GDP/\% }\end{array}$ & $\geq 30$ & $25 \sim 30$ & $20 \sim 25$ & $15 \sim 20$ & $0 \sim 15$ \\
\hline $\begin{array}{c}\text { Government R\&D investment acco } \\
\text { unted for GDP /\% }\end{array}$ & $0 \sim 0.25$ & $0.25 \sim 0.5$ & $0.5 \sim 1$ & $1 \sim 2$ & $\geq 2$ \\
\hline fompactness of urban spatial & $0 \sim 1$ & $1 \sim 1.5$ & $1.5 \sim 3$ & $3 \sim 6$ & $\geq 6$ \\
\hline form & $0 \sim 10$ & $10 \sim 20$ & $20 \sim 30$ & $30 \sim 40$ & $40 \sim 100$ \\
\hline Trban green coverage rate/\% & Worst & Worse & General & Better & Best \\
\hline Mixed land use efficiency & Least & Less & General & More & Most \\
\hline Per capita housing area & $0 \sim 10$ & $10 \sim 20$ & $20 \sim 40$ & $40 \sim 60$ & $\geq 60$ \\
\hline Coverage of walkable communities & Least & Less & General & More & Most \\
\hline Community comprehensive score & $0 \sim 20$ & $20 \sim 40$ & $40 \sim 60$ & $60 \sim 80$ & $80 \sim 100$ \\
\hline Renewable energy utilization & $0 \sim 20$ & $20 \sim 40$ & $40 \sim 60$ & $60 \sim 80$ & $80 \sim 100$ \\
\hline Annual mean air quality index & $\geq 200$ & $100 \sim 200$ & $50 \sim 100$ & $10 \sim 50$ & $\leq 10$ \\
\hline Urban forest coverage rate & $0 \sim 10$ & $10 \sim 20$ & $20 \sim 40$ & $40 \sim 60$ & $\geq 60$ \\
\hline
\end{tabular}


In the table above, Level 1, 2, 3, 4, 5 correspond to 10, 30, 50, 70, 90 points respectively.

\subsection{Mathematical Expression for Categories.}

2.3.1 Expression for Economic Development. As is shown in Figure 1, Economic Development is determined by $A_{\alpha}, A_{\beta}, A_{\gamma}$. Their corresponding levels are $A_{1}, A_{2}, A_{3}$. According to the pairwise comparison of various indicators in the first category, the judgment matrix of the criterion layer is

$$
\left[\begin{array}{ccc}
1 & 5 & 3 \\
1 / 5 & 1 & 1 / 2 \\
1 / 3 & 2 & 1
\end{array}\right]
$$

The eigenvector corresponding to the largest eigenvalue of the matrix is $0.9288,0.1747,0.3288$. The consistency test is carried out as follows:

(i) Consistency Index (CI)

The result of calculation: $C I=0.00185$.

$$
C I=\frac{\lambda_{\max }-n}{n-1}
$$

(ii) In the case $N=3$, the average random consistency index $R I=0.58$.

(iii) Consistency Ratio (CR)

$$
C=\frac{C I}{R I}
$$

The result of calculation: $C R=0.00318966$.

The consistency of the judgment matrix is acceptable, for $C R<0.1$.

The expression for Economic Development is

$$
A=0.9288 A_{1}+0.1747 A_{2}+0.3288 A_{3}
$$

\subsubsection{Expression for Urban Construction.}

As is shown in Figure 1, Urban Construction is determined by $B_{\alpha}, B_{\beta}, B_{\gamma}, B_{\delta}$. Their corresponding levels are $B_{1}, B_{2}, B_{3}, B_{4}$. According to the pairwise comparison of various indicators in the second category, the judgment matrix of the criterion layer is

$$
\left[\begin{array}{cccc}
1 & 3 & 5 & 2 \\
1 / 3 & 1 & 2 & 1 \\
1 / 5 & 1 / 2 & 1 & 1 / 3 \\
1 / 2 & 1 & 3 & 1
\end{array}\right]
$$

The eigenvector corresponding to the largest eigenvalue of the matrix is $0.8425,0.3267,0.1538$, 0.3998. The consistency test is carried out as follows:

(i) Consistency Index (CI)

$$
C I=\frac{\lambda_{\max }-n}{n-1}
$$

The result of calculation: $C I=0.0082667$.

(ii) In the case $N=4$, the average random consistency index $R I=0.90$.

(iii) Consistency Ratio (CR) 


$$
R=\frac{C I}{R I}
$$

The result of calculation: $C R=0.00918519$.

The consistency of the judgment matrix is acceptable, for $C R<0.1$.

The expression for Economic Development is

$$
B=0.8425 B_{1}+0.3267 B_{2}+0.1538 B_{3}+0.3998 B_{4}
$$

\subsubsection{Expression for Residents' Felicity Index.}

As is shown in Figure 1, Residents' Felicity Index is determined by $C_{\alpha}, C_{\beta}, C_{\gamma}$. Their corresponding levels are $C_{1}, C_{2}, C_{3}$. According to the pairwise comparison of various indicators in the third category, the judgment matrix of the criterion layer is

$$
\left[\begin{array}{ccc}
1 & 3 & 1 / 2 \\
1 / 3 & 1 & 1 / 5 \\
2 & 5 & 1
\end{array}\right]
$$

The eigenvector corresponding to the largest eigenvalue of the matrix is $0.4629,0.1640,0.8700$. The consistency test is carried out as follows:

(i) Consistency Index (CI)

$$
C I=\frac{\lambda_{\max }-n}{n-1}
$$

The result of calculation: $C I=0.00185$.

(ii) In the case $N=3$, the average random consistency index $R I=0.58$.

(iii) Consistency Ratio (CR)

$$
C R=\frac{C I}{R I}
$$

The result of calculation: $C R=0.00318966$.

The consistency of the judgment matrix is acceptable, for $C R<0.1$.

The expression for Economic Development is

$$
C=0.4629 C_{1}+0.1640 C_{2}+0.8700 C_{3}
$$

\subsubsection{Expression for Environmental Sustainability.}

As is shown in Figure 1, Environmental Sustainability is determined by $D_{\alpha}, D_{\beta}, D_{\gamma}$. Their corresponding levels are $D_{1}, D_{2}, D_{3}$. According to the pairwise comparison of various indicators in the fourth category, the judgment matrix of the criterion layer is

$$
\left[\begin{array}{ccc}
1 & 3 & 5 \\
1 / 3 & 1 & 2 \\
1 / 5 & 1 / 2 & 1
\end{array}\right]
$$

The eigenvector corresponding to the largest eigenvalue of the matrix is $0.9281,0.3288,0.1747$. The consistency test is carried out as follows:

(i) Consistency Index (CI)

$$
C I=\frac{\lambda_{\max }-n}{n-1}
$$

The result of calculation: $C I=0.00185$.

(ii) In the case $N=3$, the average random consistency index $R I=0.58$.

(iii) Consistency Ratio (CR) 


$$
C R=\frac{C I}{R I}
$$

The result of calculation: $C R=0.00318966$.

The consistency of the judgment matrix is acceptable, for $C R<0.1$.

The expression for Economic Development is

$$
D=0.9281 D_{1}+0.3288 D_{2}+0.1747 D_{3}
$$

\subsection{The Ascertain of the Weight of Each Category.}

According to relevant information and expert evaluation, we analyzed and assessed the significance of the following categories: "Economic Development", "Urban Construction”, "Residents' Felicity Index" and "Environmental Sustainability". Being unable to confirm the weight of each categories, we made an online questionnaire with a total score of 100, and 30 respondents were asked to rate each category.

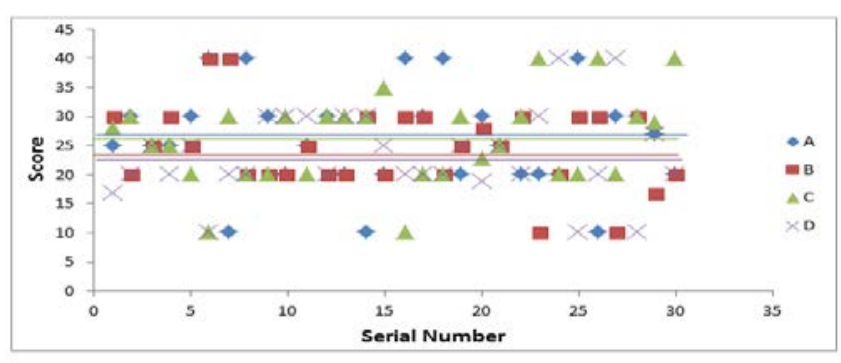

Fig.2 Prediction for weight by feedback

From the figures above, it is not difficult to see: When the quantity of feedback tends to infinity, the score of each category is likely to be close to 25. Therefore, the ultimate score of Urban Healthy Growth can be described by:

$$
\begin{gathered}
Y^{\prime}=0.25 A+0.25 B+0.25 C+0.25 D \\
Y=\frac{Y^{\prime}}{136.881} \times 100
\end{gathered}
$$

\section{Conclusions}

This paper discusses an AHP-based model to evaluate the healthy growth of a city. The specific work is as follows:

(1) Collect and divide the indicators affecting urban development into four categories.

(2) Quantify and divide each indicator into five grades of 1,2,3,4,5 by the existing data and the principles for healthy growth.

(3) Combine some complicated indicators as one indicator named Community Comprehensive Score, and conduct a survey to quantify it.

(4) Discuss the relation between the indicators to get the judgment matrix .

(5) Set up the Evaluation System of Healthy Growth.

In short, it is scientific to evaluate the urban growth situation through this model by scoring the healthy growth of a city, and the ratio of the score to 100 can be used to measure of the success of healthy growth.

\section{References}

[1] All about China. "Five evaluation indexes of urban sustainable development." China Economy. <http://www.jiemian.com/article/631163.html> (accessed 2 May, 2016) 